\title{
Propiedades físico - mecánicas de las rocas o minerales y su aplicación en el diseño de obras de ingeniería en el Callejón de Huaylas - 2013
}

Physical - mechanical properties of rocks or minerals and their application in the design of engineering works in the Callejón de Huaylas - 2013

Luis Torres Yupanqui ${ }^{1}$ y Julio Poterico Huamayalli ${ }^{1}$

\section{RESUMEN}

Detalla el procedimiento de cada uno de los ensayos, cuyo objetivo es determinar las propiedades físico-mecánicas de las rocas y minerales de una operación minera u obra civil, de acuerdo a los estándares conocidos a nivel mundial. La metodología aplicada es cuasi-experimental, por tratarse básicamente de un conjunto de ensayos que se ejecutan para cuantificar parámetros relacionados entre las variables en estudio; para su posterior análisis y evaluación, e identificar y mejorar los procedimientos de la cuantificación de dichos parámetros. A través de la evaluación de las propiedades físicas y mecánicas se determina el comportamiento de las rocas y minerales predominantes en el Callejón de Huaylas, cuya aplicación será para el diseño de obras mineras y civiles.

Palabras clave: aplicación de la Geomecánica; comportamiento de la roca; ISRM (Sociedad Internacional de Mecánica de Rocas); diseño de obras; RMR (Calidad del Macizo Rocoso).

\begin{abstract}
Detail of each one from the tests, which aim is the determination of the properties physicist - mechanics of the rocks and minerals of a mining operation or civil work, in agreement to the standards known worldwide. The applied methodology is quasi-experimental, for treating itself about a set of tests that are executed to quantify parameters related between the variables in study; for his later analysis and evaluation, to identify and to improve the procedures of the quantification of the above mentioned parameters.
\end{abstract}

Universidad Nacional Santiago Antúnez de Mayolo. Huaraz, Perú. 
Across the determination of the physical and mechanical properties there decides the behavior of the rocks and predominant minerals in Huaylas's Alley, which application they will be for the design of mining and civil works.

Keyword: application of the Geomechanic's; behavior of rock; ISRM (International Society of Rock Mechanics); design of works; RMR (Rock Mass Rating).

\section{INTRODUCCIÓN}

La minería es sin duda una de las actividades de más alto riesgo que el hombre realiza. Las estadísticas indican que la causa más frecuente de los accidentes en el interior de mina es por caída de las rocas. Según las estadísticas de los accidentes fatales ocurridos en los últimos años, aproximadamente el 23\% de estos accidentes fue por desprendimiento de rocas. Si a esta cifra le añadimos el 13\% de accidentes originados por derrumbes, deslizamientos, soplado de mineral o escombros, la segunda causa de muerte en minería, es más de 36\% de fatalidades relacionadas con la inestabilidad de las rocas (Acosta, 2007).

En obras civiles, como es la construcción de presas, diques carreteras, túneles, entre otros, el desconocimiento de la ciencia de la mecánica de rocas, que es definido como el comportamiento mecánico de las rocas y el macizo rocoso; no son tomados en cuenta los parámetros de las propiedades físico - mecánicas de las rocas, ya que los diseños de estas obras se ejecutan a través de los parámetros cuantificados por la mecánica de suelos, que esta estandarizado por el ASTM (American Society For Testing Materials), (Torres, 2004).

En nuestra Región Ancash, se pudo apreciar el colapso de muchas obras de ingeniería civil, como derrumbe de carreteras, soplado de diques como es el caso del dique la Encantada ubicado en la provincia de Pallasca, distrito de LLapo, destrucción de losas deportivas, fracturamiento de obras de construcción como edificios y casas, en la provincia de San Luis, entre otras.

En obras agrícolas relacionada a la ejecución de canales de riego, para obras de gran envergadura en nuestra región Áncash, no se tiene en cuenta las propiedades físico - mecánicas de las rocas, solo son considerados el diseño de mecánica de suelos por lo que se recomienda aplicar la ciencia de la Geomecánica.

La justificación del presente trabajo de investigación; es que en el diseño de obras de ingeniería en el Callejón de Huaylas se tome en cuenta el estudio de comportamiento de las rocas como son: rocas ígneas, rocas metamórficas, rocas sedimentarias y más aún el mineral, que son los elementos primordiales para 
la determinación de las propiedades físico-mecánicas de las rocas y minerales de una operación minera u obra civil, con cuyos resultados de los parámetros cuantificados se diseñaran las obras de ingeniería en nuestro Callejón de Huaylas.

\section{MATERIALES Y MÉTODOS}

El estudio se realizó en las obras mineras, civiles y agrícolas dentro del Callejón de Huaylas, en cada una de las cuales se realizó los ensayos de propiedades físicas y mecánicas de cada una de las muestras rocosas y/o minerales, para lo cual se hizo uso de los siguientes equipos:

$\checkmark$ Sonda Sacatestigos.

$\checkmark$ Cortadora de Disco Diamantino.

$\checkmark$ Balanza Digital.

$\checkmark$ Horno o Estufa.

$\checkmark$ Martillo Schmidt de Dureza.

$\checkmark$ Calibradores.

$\checkmark$ Equipo de Carga Puntual.

En cuanto a la metodología, se basó en los siguientes tipos de estudio:

a. El desarrollo de esta investigación es cuasi-experimental, por tratarse básicamente de un conjunto de ensayos que se ejecutan para cuantificar parámetros de las propiedades físicas y mecánicas de las rocas o minerales; para su posterior análisis y evaluación para identificar y mejorar los procedimientos de la cuantificación de dichos parámetros (Hernández, Fernández, Baptista, 2010).

b. Según el Nivel de la Investigación es descriptiva ya que se enfoca en describir todos los procedimientos para la ejecución de los ensayos, es decir es la descripción con mayor precisión de cada uno de los ensayos ejecutados (Bhappu, 1995)

Se tomó en cuenta los Estándares del ISRM ${ }^{2}$ (Society International For Rock Mechanic's), así como también el ASTM ${ }^{3}$ (American Society For Testing and Materials); respetando las características socio-culturales y geográficas, para el diseño, construcción y operatividad de las obras de ingeniería ubicadas en

2 Society International For Rock Mechanic's, (1979). Bieniawski, pág. 469.

3 American Society For Testing and Materials, (1974). Swanson, pág 105. 
la área de estudio del trabajo de investigación y en su entorno operacional, así como la legislación, normas y acuerdos internacionales en términos de respeto a la biodiversidad y uso de metodologías extractivas.

Siguiendo los cánones de la ética profesional y de una investigación responsable, el equipo actuó de manera profesional que garantice la idoneidad en el desarrollo del trabajo de investigación.

\section{RESULTADOS}

Los resultados de las propiedades físico - mecánicas de las rocas ígneas del Callejón de Huaylas se puede apreciar en la tabla 1.

Tabla 1. Propiedades físico - mecánicas de rocas ígneas

\begin{tabular}{|c|c|c|c|c|c|}
\hline \multirow{2}{*}{$\begin{array}{c}\text { MUESTRA } \\
\text { ROCOSA }\end{array}$} & \multicolumn{4}{|c|}{ PROPIEDADES FÍSICAS } & \multirow{2}{*}{$\begin{array}{c}\text { PROPIEDAD } \\
\text { MECÁNICA } \\
\text { Resistencia } \\
\text { Compresiva } \sigma_{c} \\
\mathrm{MPa}\end{array}$} \\
\hline & $\begin{array}{c}\text { Densidad } \\
\mathrm{gr} / \mathrm{cm}^{3}\end{array}$ & $\begin{array}{l}\text { P.E.A. } \\
\mathrm{KN} / \mathrm{m}^{3}\end{array}$ & $\begin{array}{l}\text { P.A. } \\
\%\end{array}$ & $\begin{array}{c}\text { Absorción } \\
\%\end{array}$ & \\
\hline ANDESITA & 2.93 & 28.74 & 2.54 & 0.87 & 239.28 \\
\hline ANDESITA & 2.87 & 28.15 & 4.31 & 1.50 & 85.65 \\
\hline GRANODIORITA & 3.01 & 29.53 & 3.44 & 1.14 & 91.24 \\
\hline ANDESITA & 2.72 & 26.68 & 3.88 & 1.43 & 120.82 \\
\hline ANDESITA & 2.61 & 25.60 & 3.33 & 1.28 & 57.03 \\
\hline DACITA & 2.81 & 27.55 & 0.50 & 0.18 & 255.53 \\
\hline GRANITO & 2.25 & 22.10 & 2.00 & 0.89 & 150.57 \\
\hline ANDESITA & 2.62 & 25.72 & 0.5 & 0.19 & 245.12 \\
\hline SKARN & 3.1 & 30.41 & 3.68 & 1.19 & 93.38 \\
\hline GRANODIORITA & 2.37 & 23.23 & 4.12 & 1.74 & 103.33 \\
\hline GRANODIORITA & 2.44 & 23.92 & 2.56 & 1.05 & 111.94 \\
\hline ANDESITA & 2.80 & 27.43 & 3.65 & 1.31 & 59.16 \\
\hline ANDESITA & 2.37 & 23.25 & 6.25 & 2.64 & 63.75 \\
\hline ANDESITA & 2.30 & 22.56 & 5.38 & 2.34 & 65.76 \\
\hline
\end{tabular}

De acuerdo a los resultados obtenidos de las rocas ígneas, se han obtenido los siguientes rangos, que se muestran en la tabla 2. 
Propiedades físico - mecánicas de las rocas o minerales y su aplicación en el diseño de obras de ingeniería|

Tabla 2. Rangos de las rocas ígneas

\begin{tabular}{|c|c|c|c|c|c|}
\hline \multirow[b]{2}{*}{$\begin{array}{c}\text { MUESTRA } \\
\text { ROCOSA }\end{array}$} & \multicolumn{4}{|c|}{ PROPIEDADES FÍSICAS } & \multirow{2}{*}{$\begin{array}{l}\text { PROPIEDAD } \\
\text { MECÁNICA } \\
\text { Resistencia } \\
\text { Compresiva } \sigma_{c} \\
\mathrm{MPa}\end{array}$} \\
\hline & $\begin{array}{l}\text { Densidad } \\
\mathrm{gr} / \mathrm{cm}^{3}\end{array}$ & $\begin{array}{l}\text { P.E.A. } \\
\mathrm{KN} / \mathrm{m}^{3}\end{array}$ & $\begin{array}{l}\text { P.A. } \\
\%\end{array}$ & $\begin{array}{c}\text { Absorción } \\
\%\end{array}$ & \\
\hline ANDESITA & $2.30-2.93$ & $22.56-28.74$ & $2.54-5.38$ & $0.87-2.34$ & $65.76-239.28$ \\
\hline GRANODIORITA & $2.37-3.01$ & $23.23-29.53$ & $3.44-4.12$ & $1.14-1.74$ & $91.24-111.94$ \\
\hline DACITA & 2.81 & 27.55 & 0.50 & 0.18 & 255.53 \\
\hline GRANITO & 2.25 & 22.10 & 2.00 & 0.89 & 150.57 \\
\hline SKARN & 3.1 & 30.41 & 3.68 & 1.19 & 93.38 \\
\hline
\end{tabular}

Los resultados de las propiedades físico - mecánicas de las rocas metamórficas del Callejón de Huaylas se puede apreciar en la tabla 3.

Tabla 3. Propiedades físico - mecánicas de rocas metamórficas

\begin{tabular}{|c|c|c|c|c|c|}
\hline \multirow[b]{2}{*}{$\begin{array}{c}\text { MUESTRA } \\
\text { ROCOSA }\end{array}$} & \multicolumn{4}{|c|}{ PROPIEDADES FÍSICAS } & \multirow{2}{*}{$\begin{array}{l}\text { PROPIEDAD } \\
\text { MECÁNICA } \\
\text { Resistencia } \\
\text { Compresiva } \sigma_{c} \\
\text { MPa } *\end{array}$} \\
\hline & $\begin{array}{c}\text { Densidad } \\
\mathrm{gr} / \mathrm{cm}^{3}\end{array}$ & $\begin{array}{l}\text { P.E.A. } \\
\mathrm{KN} / \mathrm{m}^{3}\end{array}$ & $\begin{array}{c}\text { P.A. } \\
\%\end{array}$ & $\begin{array}{c}\text { Absorción } \\
\%\end{array}$ & \\
\hline CUARCITA & 2.65 & 26.00 & 4.06 & 1.53 & 63.24 \\
\hline PIZARRA & 2.54 & 24.92 & 1.49 & 0.59 & 126.05 \\
\hline PIZARRA & 2.69 & 26.39 & 1.09 & 0.41 & 187.48 \\
\hline CUARCITA & 2.82 & 27.66 & 3.50 & 1.24 & 160.85 \\
\hline CUARCITA & 2.51 & 24.66 & 2.73 & 1.09 & 311.95 \\
\hline CUARCITA & 2.63 & 25.77 & 1.19 & 0.45 & 157.61 \\
\hline CUARCITA & 2.52 & 24.76 & 0.97 & 0.38 & 89.98 \\
\hline CUARCITA & 2.72 & 26.70 & 1.35 & 0.50 & 66.56 \\
\hline HORNFELS & 2.73 & 26.78 & 2.41 & 0.88 & 387.85 \\
\hline
\end{tabular}

De acuerdo a los resultados obtenidos de las rocas metamórficas, se han obtenido los siguientes rangos, que se muestran en la tabla 4 . 
Tabla 4. Rangos de las rocas metamórficas

\begin{tabular}{|c|c|c|c|c|c|}
\hline \multirow{2}{*}{$\begin{array}{c}\text { MUESTRA } \\
\text { ROCOSA }\end{array}$} & \multicolumn{4}{|c|}{ PROPIEDADES FÍSICAS } & \multirow{2}{*}{$\begin{array}{c}\text { PROPIEDAD } \\
\text { MECÁNICA } \\
\text { Resistencia } \\
\text { Compresiva } \sigma_{c} \\
\mathrm{MPa}^{*}\end{array}$} \\
\hline & $\begin{array}{l}\text { Densidad } \\
\mathrm{gr} / \mathrm{cm}^{3}\end{array}$ & $\begin{array}{l}\text { P.E.A. } \\
\text { KN/m³ }\end{array}$ & $\begin{array}{l}\text { P.A. } \\
\%\end{array}$ & $\begin{array}{c}\text { Absorción } \\
\%\end{array}$ & \\
\hline CUARCITA & $2.51-2.82$ & $24.66-27.66$ & $0.37-4.06$ & $0.38-1.53$ & 63.24-311.95 \\
\hline PIZARRA & $2.54-2.69$ & $24.92-6.39$ & $1.09-1.49$ & $0.41-0.59$ & $126.05-187.48$ \\
\hline HORNFELS & 2.73 & 26.78 & 2.41 & 0.88 & 387.85 \\
\hline
\end{tabular}

Los resultados de las propiedades físico - mecánicas de las rocas sedimentarias del Callejón de Huaylas se puede apreciar en la tabla 5.

Tabla 5. Propiedades físico - mecánicas de rocas sedimentarias

\begin{tabular}{|c|c|c|c|c|c|}
\hline \multirow{2}{*}{$\begin{array}{c}\text { MUESTRA } \\
\text { ROCOSA }\end{array}$} & \multicolumn{4}{|c|}{ PROPIEDADES FÍSICAS } & \multirow{2}{*}{$\begin{array}{c}\text { PROPIEDAD } \\
\text { MECÁNICA } \\
\text { Resistencia } \\
\text { Compresiva } \sigma \\
\text { MPa } *\end{array}$} \\
\hline & $\begin{array}{l}\text { Densidad } \\
\mathrm{gr} / \mathrm{cm}^{3}\end{array}$ & $\begin{array}{l}\text { P.E.A. } \\
\text { KN/m } 3\end{array}$ & $\begin{array}{c}\text { P.A. } \\
\%\end{array}$ & $\begin{array}{c}\text { Absorción } \\
\% \\
\end{array}$ & \\
\hline CALIZA & 2.63 & 25.80 & 1.45 & 0.55 & 240.17 \\
\hline CALIZA & 2.69 & 26.37 & 1.00 & 0.37 & 86.22 \\
\hline CALIZA & 3.09 & 30.35 & 1.84 & 0.59 & 96.05 \\
\hline $\begin{array}{c}\text { CALIZA } \\
\text { SILICIFICADA }\end{array}$ & 2.45 & 24.03 & 1.00 & 0.41 & 254.27 \\
\hline LUTITA & 2.74 & 26.84 & 2.58 & 0.94 & 222.37 \\
\hline CALIZA & 2.47 & 24.22 & 2.32 & 0.96 & 92.71 \\
\hline CALIZA & 3.20 & 31.39 & 2.03 & 0.63 & 239.67 \\
\hline LUTITA & 2.88 & 28.25 & 6.35 & 2.20 & 35.22 \\
\hline CALIZA & 2.78 & 27.27 & 1.59 & 0.57 & 227.49 \\
\hline ARENISCA & 2.88 & 28.24 & 1.28 & 0.74 & 303.76 \\
\hline ARENISCA & 2.86 & 28.04 & 1.58 & 0.55 & 248.02 \\
\hline CALIZA & 2.52 & 24.72 & 5.00 & 1.98 & 127.78 \\
\hline CALIZA & 2.52 & 24.72 & 1.05 & 0.42 & 70.45 \\
\hline CALIZA & 2.52 & 24.72 & 1.05 & 0.42 & 70.45 \\
\hline
\end{tabular}


De acuerdo a los resultados obtenidos de las rocas sedimentarias, se han obtenido los siguientes rangos, que se muestran en la tabla 6 .

Tabla 6. Rango de las rocas sedimentarias

\begin{tabular}{|c|c|c|c|c|c|}
\hline \multirow[b]{2}{*}{$\begin{array}{l}\text { MUESTRA } \\
\text { ROCOSA }\end{array}$} & \multicolumn{4}{|c|}{ PROPIEDADES FÍSICAS } & \multirow{2}{*}{$\begin{array}{l}\text { PROPIEDAD } \\
\text { MECÁNICA } \\
\text { Resistencia } \\
\text { Compresiva } \sigma_{c} \\
\mathrm{MPa}^{*}\end{array}$} \\
\hline & $\begin{array}{c}\text { Densidad } \\
\mathrm{gr} / \mathrm{cm}^{3}\end{array}$ & $\begin{array}{l}\text { P.E.A. } \\
\mathrm{KN} / \mathrm{m}^{3}\end{array}$ & $\begin{array}{l}\text { P.A. } \\
\%\end{array}$ & $\begin{array}{c}\text { Absorción } \\
\%\end{array}$ & \\
\hline CALIZA & $2.47-3.20$ & $24.22-30.35$ & $1.00-5.00$ & $0.37-1.98$ & $70.45-239.67$ \\
\hline $\begin{array}{c}\text { CALIZA } \\
\text { SILICIFICADA }\end{array}$ & 2.45 & 24.03 & 1.00 & 0.41 & 254.27 \\
\hline LUTITA & $2.74-2.88$ & $26.84-28.25$ & $2.58-6.35$ & $0.94-2.20$ & $35.22-222.37$ \\
\hline ARENISCA & $2.86-2.88$ & $28.04-28.24$ & $1.28-1.58$ & $0.55-0.74$ & $248.02-303.76$ \\
\hline
\end{tabular}

\section{DISCUSIÓN}

Las propiedades físico mecánicas de las rocas y minerales en el Callejón de Huaylas de la región Áncash, constituye en la actualidad un problema, por su aplicación en el diseño de obras de ingeniería, que no son tomados en cuenta por el desconocimiento de los parámetros del comportamiento de las rocas y minerales.

Durante la ejecución de los ensayos para la determinación de las propiedades físicas como: La densidad, la porosidad aparente, la absorción, el peso específico aparente y la resistencia compresiva de la roca y mineral que es aplicado en el diseño de obras de Ingeniería, en el Callejón de Huaylas; se tomaron en cuenta los procedimientos del estándar del ISRM (Society International For Rock Mechanic’s) Sociedad Internacional de Mecánica de Rocas.

Los resultados nos muestran que actualmente en las obras de ingeniería (minas, civil y agrícolas) se va tomando mayor importancia, la aplicación de la ciencia de la Geomecánica a través de la cuantificación de las propiedades físico - mecánicas de la rocas del Callejón de Huaylas, ya que de acuerdo a las propiedades de las rocas y minerales a nivel mundial son semejantes su comportamiento mecánico.

\section{CONCLUSIONES}

Las propiedades físico - mecánicas de las rocas y mineral más representativas del Callejón de Huaylas, tienen los siguientes rangos para su aplicación: 
ROCAS ÍGNEAS: La Resistencia Compresiva de acuerdo a Deere And Miller es: Andesita: resistencia media a resistencia muy alta; Granodiorita: resistencia media a resistencia alta; Dacita: resistencia muy alta; Granito: resistencia alta y Skarn: resistencia media.

ROCAS METAMORFICAS: La Resistencia Compresiva de acuerdo a Deere And Miller es: Cuarcita: resistencia alta a resistencia muy alta; Pizarra: resistencia alta y Hornfels: resistencia muy alta.

ROCAS SEDIMENTARIAS: La Resistencia Compresiva de acuerdo a Deere And Miller es: Caliza: resistencia media a resistencia muy alta; Lutita: resistencia baja resistencia muy alta; Arenisca: resistencia muy alta y Caliza silicificada: resistencia muy alta.

\section{AGRADECIMIENTOS}

Se agradece a la Universidad Nacional Santiago Antúnez de Mayolo, en particular a la Facultad de Ingeniería de Minas Geología y Metalurgia por apoyarnos en la data básica de los ensayos ejecutados en el Laboratorio de Mecánica de Rocas.

\section{REFERENCIAS BIBLIOGRÁFICAS}

Acosta, Jorge. 2007. Aplicación de las Inclusiones Fluidas en la búsqueda de yacimientos de Cobre, ejemplo: Porfido de cobre Lara. Ayacucho: Oveja negra.

Bhappu, Rosan. 1995. The sequential copper analysis Method-Geological, Mineralogical and Metallurgical implications. Denver, Colorado: SME Meeting.

Hernandez, Roberto; Fernandez, Carlos; Baptista, Pilar. 2010. Metodología de la Investigación. México: McGraw Hill.

Torres, Luis 2004. Manual de la Determinación de Propiedades Físico - Mecánicas de las Rocas y Monitoreo de la Masa Rocosa. Huaraz.

Presentado: $16 / 01 / 2017$

Aceptado: 01/05/2017

\section{Correspondencia}

Luis Alberto Torres Yupanqui

latorresy@gmail.com 